\title{
Investigating Factors Associated with Vaccine Hesitancy in Makkah, KSA
}

\author{
Rana Albarakati ${ }^{1}$, Lujain Almatrafi ${ }^{1}$, Gaidaa Fatta ${ }^{1}$, Batool Fatani1 ${ }^{1}$ Yosra Alhindi ${ }^{*}$ \\ ${ }^{1}$ Faculty of Pharmacy, University of Umm Al-Qura, Makkah, KSA \\ ${ }^{2}$ Clinical Pharmacy Department, Pharmacy College, Umm Al-Qura University, Makkah, KSA \\ Email: *yzhindi@uqu.edu.sa
}

How to cite this paper: Albarakati, R., Almatrafi, L., Fatta, G., Fatani, B. and Alhindi, Y. (2019) Investigating Factors Associated with Vaccine Hesitancy in Makkah, KSA. World Journal of Vaccines, 9, 37-48. https://doi.org/10.4236/wjv.2019.92003

Received: February 13, 2019

Accepted: April 1, 2019

Published: April 4, 2019

Copyright (c) 2019 by author(s) and Scientific Research Publishing Inc. This work is licensed under the Creative Commons Attribution International License (CC BY 4.0).

http://creativecommons.org/licenses/by/4.0/

\section{cc) (7) Open Access}

\begin{abstract}
Introduction: Vaccination provides health and economic benefits to the individual and to society. In Saudi Arabia, public support for immunisation programmes is generally high and well received. However, the benefits of vaccines are often not fully known or appreciated. When public discussion on vaccine safety, quality or efficacy occurs, very often misinformation creeps into the debate through the internet and other media sources, significantly weakening immunisation programmes. Vaccine hesitancy is a concept frequently used in the discourse around vaccine acceptance. This study reflects on the factors associated with vaccine hesitancy, through a study focusing on knowledge, attitudes and beliefs among parents of young children. Methods: A cross-sectional study was carried out in seven primary schools in Makkah city, Saud Arabia. Data were collected in 2017 through a computer based-survey administered to 100 parents with children aged between 2 months and 17 years of age. Questionnaire responses were analysed using Minitab. Results: Many parents (with a total of 292 children) agreed on the importance of vaccinations, to protect their children's health and to prevent the spread of diseases in the community. Most parents $(68.7 \%, \mathrm{P}<0.05)$ reported their child had received all recommended vaccines. Approximately one third $(31.3 \%, \mathrm{P}<$ $0.05)$ of parents indicated their hesitation in having their child vaccinated. The lack of vaccines in primary care centres, fear of adverse events and vaccine safety were the reasons most frequently mentioned by these vaccine-hesitant parents. In addition, some parents believed vaccines had become a business and that profits were more important than their children's safety. Other parents complained about the increasingly "crowded" vaccination schedules. A minority of parents believe that vaccines did not prevent the spread of disease in the community $(\mathrm{P}<0.05)$. Discussion and Conclusions: Vaccine hesitancy is a common problem in Makkah, Saudi Arabia. Further research will be needed to better understand when, how and why these beliefs are formed.
\end{abstract}




\section{Keywords}

Vaccine, Hesitancy, Parents, Children

\section{Introduction}

Vaccination programmes in Saudi Arabia achieve national goals by protecting society against transitional diseases (hepatitis B, Tuberculosis and Rotavirus) [1]. The programmes respond to global requirements agreed by countries of the world, through recommendations from the World Health Organisation, to eradicate polio, measles, and tetanus and prevent hepatitis $\mathrm{B}$, meningitis and influenza outbreaks [2]. The national vaccination programme ensures most of the population has received vaccines included in immunisation schedules. The term vaccine Hesitancy "refers to delay in acceptance or refusal of vaccines despite availability of vaccine services" [3].

To understand vaccines and how they function, it is important to understand the immune system. Vaccines teach our immune system how to fight and protect against foreign invaders. Infection occurs when foreign invaders, like viruses and bacteria attack and multiply in the body. Immune cells or white blood cells fight and protect the body from these invaders. Immune cells are also called lymphocytes and mainly consist of B-lymphocytes, T-lymphocytes and macrophages [4].

A vaccine is a biological preparation made from either a whole microbe or parts of the microbe, such as toxins or surface proteins. Although vaccines contain agents that resemble disease-causing microorganisms, they do not cause illness because these components are made from weakened or dead microbes. As the body builds its immunity, minor symptoms, such as slight fever, are normal and should be expected [5].

Vaccines initiate their action by mimicking infection. Even though this infection does not develop to disease, it leads to the production of T-lymphocytes and antibodies. So, in the future, if the body is attacked by the same microbe, it will remember how to fight back, thanks to memory T-lymphocytes. However, after vaccination, it normally takes several days before the immune system starts producing T-lymphocytes and B-lymphocytes [5]. Therefore, if an individual becomes infected with a disease, just before or after vaccination, it is possible they could still develop the disease.

Vaccines are one of the most successful medical advances in modern times. However, individuals fear them because they do not fully understand the risks. To quash delusions and rumours about vaccines, they should be treated as any normal routine medicine [5]. This means the risk/benefits assessment of any vaccine should be communicated to and understood by all concerned.

Most vaccine-preventable illnesses are unfamiliar to parents. This means parents increasingly question the necessity of immunising their children, especially 
as no vaccine is completely free from adverse effects or complication risks. Family physicians must be aware of the risks and benefits of immunisations and provide parents with a clear picture of any issues [6].

Vaccines are different from most medicines; they are administered to large and mostly healthy populations, including infants and children, so tolerances are low for potential risks or side-effects [5]. Acceptance of vaccine side-effects depends on their frequency and severity. Acceptance may also vary with time, based on how the side-effects compare with the symptoms induced by illness [6]. It is however unacceptable for vaccines to induce significant side effects, even where the illness itself produces severe or fatal side effects. Adverse events (AEs) occurring after immunisation, regardless of whether they were caused by the vaccine or not, are "adverse events following immunisation" [7].

Our main objective in this study was to investigate the basis and prevalence of controversies surrounding vaccines in Makkah, Saudi Arabia. This was done by examining data and claims surrounding vaccine controversies.

\section{Materials and Methods}

We have conducted a descriptive cross-sectional study in seven primary schools in Makkah city, Saudi Arabia. This study was conducted during the period of January to April 2017. The schools were collected by random sampling. Sampling was stratified through the different geographical areas of Makkah city. Sample size was calculated based on the formula $(\mathrm{n}=\mathrm{Z} 1-\alpha 2 \mathrm{P}(1 \mathrm{P}) / \mathrm{d} 2)$, where $\mathrm{n}$ $=$ sample size $=256, \mathrm{Z}=$ standard normal variate $=1.96$ (at $5 \%$ type $\mathrm{I}$ error, $\mathrm{p}=$ $0.05), \mathrm{P}=$ expected proportion $=50 \%$, and $\mathrm{d}=$ precision error $=5 \%$. Additional $20 \%$ was added to cover the missing data. The total sample obtained was 292 children and a total number of 100 parents. A computer-based survey about vaccine hesitancy was given to each parent. A letter of objectives and parents consents were also given. The questionnaire required information about parental opinion on vaccines, their trust, their attitude and believes towards it. It was consisted of 25 questions was written by the authors and checked the validity using Content validity index with a score of $I-C V I=0.90$. In the inclusion criteria parent with children 0 - 12 years old were invited to participate. We excluded vaccines that was not included in the governmental vaccination scheduled such as influenza vaccine also we excluded acutely ill children. All data were collected by the authors and were processed using Minitab software (version 2018, USA). Descriptive statistics were used to describe all variables and we used t-test to compare the results with a $\mathrm{P}$ value $<0.05$.

\section{Results}

Approximately $68.7 \%$ of parents reported their child had received all recommended vaccines, but $31.3 \%$ of parents indicated a hesitation in having their child vaccinated $(\mathrm{P}<0.05)$ (Figure $1(\mathrm{a})$ ). The lack of vaccines in primary care centres (60\%), the fear of adverse events (25\%) and vaccine safety $(13.7 \%)$ were the most frequent reasons mentioned by these vaccine-hesitant parents. Addi- 
tionally, some parents $(1.2 \%)$ believed that vaccines had become a business for profit, rather than for the safety of their children. Other parents $(0.2 \%)$ complained about the increasingly "crowded" vaccination schedules. No other major concerns were mentioned by parents towards vaccination. However, a minority of parents $(\mathrm{P}<0.05)$ did not believe in the importance of vaccines in preventing the spread of diseases in the community (Figure 1(b)).

Approximately, $59.6 \%$ of parents $(\mathrm{P}<0.05)$ did not have enough information about potential adverse events following vaccination (Figure 2). This was cited as a reason, driving their hesitancy in getting their child vaccinated. The parents explain the lack of information as they did not receive any brochures, booklist or educational community sessions or even simple instructions inside the clinics to educate and teach them about the most important side effects that can face their children.

VACCINATED

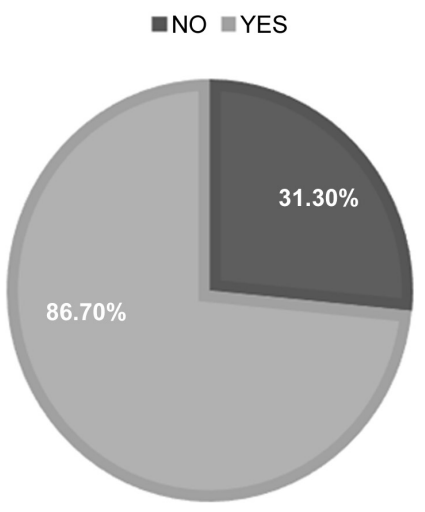

(a)

\section{BELIEVE}

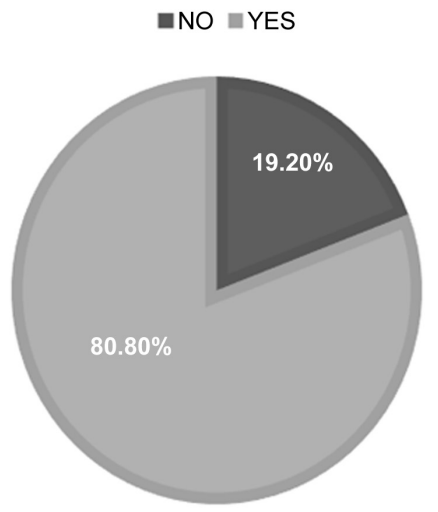

(b)

Figure 1. (a) Graph reflecting vaccinated and unvaccinated children (\%). N = 292 children, T-test: $(\mathrm{P}<0.05)$. (b) Graph reflecting how parents believe towards vaccination $(\%)$. $\mathrm{N}=100$ parents, T-test: $(\mathrm{P}<0.05)$.

\section{Knowldge of vaccination side effects}

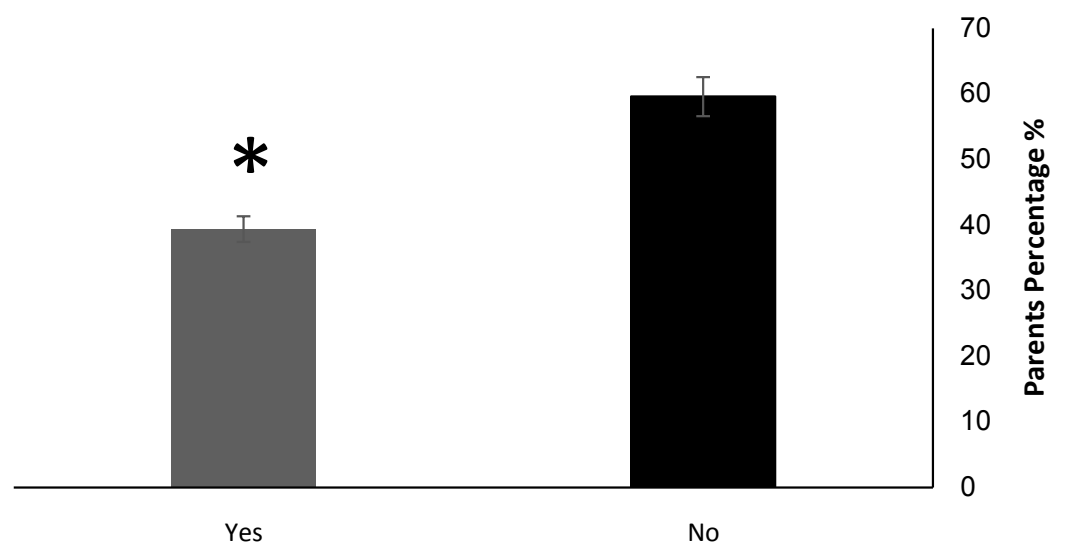

Figure 2. Graph showing parents' knowledge of vaccination adverse effects, T-test: $(\mathrm{P}<$ 0.05). Yes: have knowledge; No: don't know. $\mathrm{N}=100$ parents. 


\section{Discussion}

The term "vaccine hesitancy" has only recently emerged and has been used by many anti-vaccination movements. The evidence proving the benefits of immunisation is overwhelming and has been established through a growing number of clinical trials [8]. Vaccinating has proven to be one of the most successful and cost-effective interventions in improving modern era health outcomes [9]. Regardless of vaccine hesitancy, vaccinating is still the best thing for a parent to do for their child. To better address vaccine hesitancy within a specific country or a population, we must first understand the extent of the problem and where it came from [8]. The aim of this study was to investigate the basis and prevalence of controversies surrounding vaccines in Makkah, Saudi Arabia and examine data and claims surrounding the vaccine controversy, vaccine hesitancy.

Saudi Arabia has greatly improved the national immunisation programme through seasonal immunisation campaigns, and awareness campaigns in schools and hospitals. However, primary healthcare centres need more attention and focus. They are the main vaccine providers and any stock shortages will mean children will not receive their appropriate vaccines in a timely manner. This could lead to risks in child health, thereby affecting immunisation rates [5].

Herd immunity is the most effective way of avoiding and decreasing incidence rates of disease [10]. It is established when $95 \%$ of the population has been vaccinated against a specific disease. All humans and their immune systems differ; therefore, every immune system will react differently to the vaccine, in the same manner but still individually different. Therefore, creating a herd immunity may be problematic, if for example a virus mutates into a different form, one that the body cannot fight off [11].

In this research, we addressed several hypotheses that potentially risk the lives of children. While these hypotheses are highly questionable and controversial, parents still believe in them and hold back on vaccinating their children. Their perspectives need to be enlightened.

The first hypothesis claims that the combination measles-mumps-rubella vaccine causes autism by damaging the intestinal lining, allowing the expression of encephalopathic proteins, towards the brain [12]. While there is no data to support this mechanism, several epidemiological studies have emerged to correct some parents' view on the MMR vaccine. In the United Kingdom, researchers evaluated 489 autistic children born between 1979 and 1992; these children were selected from computerised health records. Although autism cases increased, no changes were observed to suggest that autism rates changed after the MMR vaccine was introduced in 1987. Furthermore, researchers did not observe increases in autism diagnoses, concomitant with the MMR vaccine [8] [13].

In California, researchers compared the annual vaccination rates of the MMR vaccine in kindergarten students with the number of autism cases, between 1980 and 1994. As observed in the United Kingdom, the increase in autism diagnoses 
was not related to MMR vaccination rates [5] [14]. In Canada, researchers estimated the prevalence of autism related to MMR, in 27,749 children. The study showed an increase in autism rates with a marked drop in MMR vaccination rates [9]. In a Danish retrospective study, researchers specified the vaccination status and autism diagnoses in 537,303 children, born between 1991 and 1998. They observed no differences in autism risk among children who received and did not receive the MMR vaccine [15].

The second hypothesis states that thimerosal, an ethyl mercury-containing preservative in some vaccines, is toxic to the CNS. Autism is not acquired with the thimerosal vaccine; seven descriptive and observational studies were performed, confirming no relation between the thimerosal vaccine and autism. In Denmark, researchers conducted a comparative study of autism in children who 1) received $200 \mathrm{mg}$ thimerosal between 1961 and 1970, 2) received $125 \mu \mathrm{g}$ thimerosal between 1970 and 1992, and 3) received $0 \mu \mathrm{g}$ thimerosal between 1992 and 2000. The study showed no relationship between thimerosal-based vaccines and autism [16] [17] [18].

In Canada, researchers grouped 27,749 children, from 55 schools, using birth dates, and estimated the prevalence of autism related to thimerosal exposure. The study showed that the highest rates of autism and other developmental disorder were in cohorts exposed to thimerosal-free vaccines [19]. In the United Kingdom, researchers evaluated immunisation records for 100,572 children born between 1988 and 1997, of which 104 had autism. No association was observed between thimerosal-based vaccines and autism diagnoses [20] [21].

The third hypothesis suggests that the simultaneous administration of multiple vaccines overwhelms or weakens the immune system. The hypothesis proposes that children may be receiving too many vaccines too soon, and that these vaccines either make the immune system immature or induce autism autoimmune responses [22]. These observations are not possible for several reasons; 1) multiple vaccinations do not alter or weaken the immune system. Vaccinated and unvaccinated children do not differ in their susceptibility to infections [23], 2) vaccines do not overwhelm the immune system. Although the immune system of infants is relatively naive, it can generate a protective response and this response can simultaneously withstand thousands of vaccines [3] [24]. Moreover, the immune responses of multiple vaccines are like individual vaccine response [13] [25], 3) autism is not an immune-mediated disease. Unlike autoimmune diseases such as multiple sclerosis, there is no evidence of immuno-activation in autistic children [16] [26]. Current data suggest that genetic variation in the neuronal system may affect synaptic development resulting in autistic behaviour [18].

Generally, most interventions intended to increase immunisation rates in this review were not dramatically different from traditional strategies [17] [26]. Interventions focused on the individual and society, with the aim of increasing vaccine knowledge and awareness. However, these interventions were not effec- 
tive in improving vaccine-acceptance, probably because most effective interventions use multi-component strategies. Additionally, most were tailored to specific populations and concerns. This is important motive in understanding the drivers of vaccines hesitancy. We must use this knowledge to support interventions to deliver optimum performances in increasing immunisation rates.

Through asking the research question and using the questionnaire data, this research has shown that most participants agreed that vaccinations protected their children's health and prevented the spread of disease in the community. Approximately $68.7 \%$ of parents reported their child had received all recommended vaccines, but $31.3 \%$ of parents hesitated to have their child vaccinated. The lack of vaccines in primary care centres, the fear of adverse events and vaccine safety were the most frequent reasons mentioned by these vaccine-hesitant parents. In addition, some parents believed that vaccines have become a business for profit, rather than a safety measure for their children. Other parents complained about the increasingly "crowded" vaccination schedules. A minority of parents did not believe in the importance of vaccines in preventing the spread of disease in the community. Our results agreed with a cross-sectional study that was done in Tabuk city; about $95 \%$ of the parents agreed that vaccination is vital for their children and their main concern were about side effects [21] [27].

Data from the questionnaire showed that parents had a good knowledge and positive attitudes on some aspects of childhood immunisations. However, more educational interventions are recommended.

\section{Study Limitations}

The questionnaire was conducted in a small geographical area in Saud Arabia (Makkah), therefore, the results cannot be generalised to all parents in the country. To address this, future research should recruit parents from different areas in Saudi Arabia.

\section{Future Strategies}

There is limited evidence guiding effective strategies in dealing with the threat of parental vaccine refusal. There is a need for appropriately designed, well executed and evaluated interventional studies to address this knowledge gap. Strategies may include:

1) Engagement of religious or other influential leaders to promote community vaccination strategies,

2) Social mobilisation,

3) Mass media,

4) Improving convenience and access to vaccination,

5) Mandating vaccinations/sanctions for non-vaccination,

6) Employing reminder and follow-up,

7) Communications training for health care professionals,

8) Non-financial incentives and 
9) Increasing vaccination knowledge and awareness.

\section{Acknowledgements}

Special thanks to Umm Al-Qura University whom approved this research by the local research committee.

\section{Data Availability}

The Data of the all the experiments and all the analysis used to support these findings of this study are available from the corresponding author upon request.

\section{Conflicts of Interest}

The authors declare that there is no conflict of interest regarding the publication of this paper.

\section{References}

[1] Fombonne, E., Zakarian, R., Bennett, A., Meng, L. and McLean-Heywood, D. (2006) Pervasive Developmental Disorders in Montreal, Quebec, Canada: Prevalence and Links with Immunizations. Pediatrics, 118, e139-e150. https://doi.org/10.1542/peds.2005-2993

[2] WHO (2018). http://www.who.int/immunization/monitoring_surveillance/data/sau.pdf

[3] Posfay-Barbe, K.M., Heininger, U., Aebi, C., Desgrandchamps, D., Vaudaux, B. and Siegrist, C.A. (2005) How Do Physicians Immunize Their Own Children? Differences among Pediatricians and Nonpediatricians. Pediatrics, 116, e623-e633.

[4] Verani, J., Baqui, A., Broome, C., Cherian, T., Cohen, C., et al. (2017) Case-Control Vaccine Effectiveness Studies: Preparation, Design, and Enrollment of Cases and Controls. Vaccine, 35, 3295-3302. https://doi.org/10.1016/j.vaccine.2017.04.037

[5] Wakefield, A.J., Murch, S.H., Anthony, A., et al. (1998) Ileal-Lymphoid-Nodular Hyperplasia, Non-Specific Colitis, and Pervasive Developmental Disorder in Children. The Lancet, 351, 637-641. https://doi.org/10.1016/S0140-6736(97)11096-0

[6] MacDonald, N. (2015) Vaccine Hesitancy: Definition, Scope and Determinants. Vaccine, 33, 4161-4164. https://doi.org/10.1016/j.vaccine.2015.04.036

[7] Miller, E. (2015) Controversies and Challenges of Vaccination: An Interview with Elizabeth Miller. BMC Medicine, 13, 131-135. https://doi.org/10.1186/s12916-015-0508-z

[8] Song, G. (2014) Understanding Public Perceptions of Benefits and Risks of Childhood Vaccinations in the United States. Risk Analysis. An International Journal, 34, 541-555. https://doi.org/10.1111/risa.12114

[9] Keelan, J., Pavri, V., Balakrishnan, R. and Wilson, K. (2010) An Analysis of the Human Papilloma Virus Vaccine Debate on MySpace Blogs. Vaccine, 28, 1535-1540. https://doi.org/10.1016/j.vaccine.2009.11.060

[10] Gerber, J. and Offit, P. (2009) Vaccines and Autism: A Tale of Shifting Hypotheses. Clinical Infectious Diseases, 48, 456-461. https://doi.org/10.1086/596476

[11] Morrow, E.M., Yoo, S.Y., Flavell, S.W., et al. (2008) Identifying Autism Loci and Genes by Tracing Recent Shared Ancestry. Science, 321, 218-223. https://doi.org/10.1126/science.1157657 
[12] Kennedy, A.M., Brown, C.J. and Gust, D.A. (2005) Vaccine Beliefs of Parents Who Oppose Compulsory Vaccination. Public Health Reports, 120, 252-258.

[13] McCormick, M.C. (2004) Immunization Safety Review: Vaccines and Autism. Institute of Medicine, Washington DC.

[14] Nelson, K.B. and Bauman, M.L. (2003) Thimerosal and Autism? Pediatrics, 111, 674-679.

[15] Farrington, C.P., Miller, E. and Taylor, B. (2001) MMR and Autism: Further Evidence against a Causal Association. Vaccine, 19, 3632-3625.

https://doi.org/10.1016/S0264-410X(01)00097-4

[16] King, G.E. and Hadler, S.C. (1994) Simultaneous Administration of Childhood Vaccines: An Important Public Health Policy that Is Safe and Efficacious. The Pediatric Infectious Disease Journal, 13, 394-407. https://doi.org/10.1097/00006454-199405000-00012

[17] Palache, A. (2011) Seasonal Influenza Vaccine Provision in 157 Countries (2004-2009) and the Potential Influence of National Public Health Policies. Vaccine, 29, 9459-9466. https://doi.org/10.1016/j.vaccine.2011.10.030

[18] Madsen, K.M., Hviid, A., Vestergaard, M., et al. (2002) A Population-Based Study of Measles, Mumps, and Rubella Vaccination and Autism. The New England Journal of Medicine, 347, 1477-1482. https://doi.org/10.1056/NEJMoa021134

[19] Offit, P.A. (2008) Vaccines and Autism Revisited-The Hannah Poling Case. The New England Journal of Medicine, 358, 2089-2091. https://doi.org/10.1056/NEJMp0802904

[20] Dales, L., Hammer, S.J. and Smith, N.J. (2001) Time Trends in Autism and in MMR Immunization Coverage in California. JAMA, 285, 1183-1185. https://doi.org/10.1001/jama.285.9.1183

[21] Saleh, W.E., et al. (2017) Evaluation of Vaccine Hesitancy in Tabuk Population, Saudi Arabia. Journal of International Medical Research, 6, 22-25.

[22] Davies, P., Chapman, S. and Leask, J. (2002) Antivaccination Activists on the World Wide Web. Archives of Disease in Childhood, 87, 22-25. https://doi.org/10.1136/adc.87.1.22

[23] Centers for Disease Control Prevention (1999) Thimerosal in Vaccines: A Joint Statement of the American Academy of Pediatrics and the Public Health Service. Morbidity and Mortality Weekly Report, 48, 563-565.

[24] Baker, J.P. (2001) Mercury, Vaccines, and Autism: One Controversy, Three Histories. American Journal of Public Health, 98, 244-253. https://doi.org/10.2105/AJPH.2007.113159

[25] Andrews, N., Miller, E., Grant, A., Stowe, J., Osborne, V. and Taylor, B. (2004) Thimerosal Exposure in Infants and Developmental Disorders: A Retrospective Cohort Study in the United Kingdom Does Not Support a Causal Association. Pediatrics, 114, 584-591. https://doi.org/10.1542/peds.2003-1177-L

[26] Black, S.B., Cherry, J.D., Shinefield, H.R., Fireman, B., Christenson, P. and Lampert, D. (1991) Apparent Decreased Risk of Invasive Bacterial Disease after Heterologous Childhood Immunization. The American Journal of Diseases of Children, 145, 746-749. https://doi.org/10.1001/archpedi.1991.02160070042019

[27] Di Pasquale, A., Bonanni, P., Garçon, N., Stanberry, L., El-Hodhod, M. and Da Silva, F.T. (2017) Vaccine Safety Evaluation: Practical Aspects in Assessing Benefits and Risks. Vaccine, 34, 6672-6680. https://doi.org/10.1016/j.vaccine.2016.10.039 


\section{Questionnaire}

1-How many children in the family?

- 1 child

- 2 children

- 3 children

- 4 children

- 5 or more...

2-Did all the children have been vaccinated?

- Yes

- No

3-If your answer in the previous question is No so how many children did not receive vaccines?

- 1 child

- 2 children

- 3 children

- 4 children

- 5 or more...

4-Can you tell us the reason why you did not vaccinate you child?

5-Mother age?

- $20-30$

- $30-40$

- 40 - 50

6-Father age?

- $20-30$

- $30-40$

- 40 - 50

7-Educational level for the mother?

- Primary

- Secondary

- High school

- Bachelor/post graduate

8-Educational level for the father?

- Primary

- Secondary

- High school

- Bachelor/post graduate

9-Did you have any medical condition, as diabetes of gestational diabetes?

- Yes

- No

10-While you were pregnant or during delivery did you suffer from any illness or complications? 
- Yes

- No

11-Did your unvaccinated child have any illness or chronic disorder?

- Yes

- No

12-If your child have any illness what is it?

13-Do you believe that vaccines protect from infectious diseases?

- Yes

- No

14-Did you read or herd any subject concerns vaccination made you change your mind about vaccinating your children?

- Yes

- No

15-Did any thing happened made you untrusty vaccination? If yes what is it?

- Yes

- No 16-Did you know any family or parents did not vaccinate for religiousreasons?

- Yes

- No

17-Do you trust that ministry of health provide what is best for your child vaccination?

- Yes

- No

18-Is the distention to your local primary health care long or is it crowded that's why you refuse vaccination?

- Yes

- No

19-Do you think there is pressure from vaccination manufacturing companies to ministry of health?

- Yes

- No

20-Do you think these factories are safe?

- Yes

- No 21-Does the information provided to you are they enough?

- Yes

- No

22-Are you aware and familiar with the possible side effects?

- Yes

- No 
23-Did you ever know any one was harmed by vaccination?

- Yes

- No

24-Do you think the age in the governmental vaccinations chedule is appropriate?

- Yes

- No

25-Do you believe that vaccination prevented infectious disease successfully?

Thank you!

End of questions. 